\title{
Field enhancement in subnanometer metallic gaps
}

\author{
A. García-Martín, ${ }^{1}$ D. R. Ward, ${ }^{2}$ D. Natelson, ${ }^{2,3}$ and J. C. Cuevas ${ }^{4}$ \\ ${ }^{1}$ IMM-Instituto de Microelectrónica de Madrid (CNM-CSIC), Isaac Newton 8, PTM, Tres Cantos, E-28760 Madrid, Spain \\ ${ }^{2}$ Department of Physics and Astronomy, Rice University, 6100 Main St., Houston, Texas 77005, USA \\ ${ }^{3}$ Department of Electrical and Computer Engineering, Rice University, 6100 Main St., Houston, Texas 77005, USA \\ ${ }^{4}$ Departamento de Física Teórica de la Materia Condensada, Universidad Autónoma de Madrid, E-28049 Madrid, Spain
}

(Received 2 March 2011; published 19 May 2011)

\begin{abstract}
Motivated by recent experiments [D. R. Ward et al., Nat. Nanotechnol. 5, 732 (2010)], we present here a theoretical analysis of the optical response of sharp gold electrodes separated by a subnanometer gap. In particular, we have used classical finite difference time domain simulations to investigate the electric-field distribution in these nanojunctions upon illumination. Our results show a strong confinement of the field within the gap region, resulting in a large enhancement compared to the incident field. Enhancement factors exceeding $10^{3}$ are found for interelectrode distances on the order of a few angstrom, which are fully compatible with the experimental findings. Such huge enhancements originate from the coupling of the incident light to the evanescent field of hybrid plasmons involving charge density oscillations in both electrodes.
\end{abstract}

DOI: 10.1103/PhysRevB.83.193404

PACS number(s): 78.67.Uh, 73.20.Mf, 73.21.Hb

Introduction. The study of the optical response of metallic nanostructures is revealing fascinating physics. ${ }^{1,2}$ Special attention is being paid to the analysis of the so-called optical and infrared gap nanoantennas, which consist of adjacent metallic segments, like nanorods, separated by a nanoscale gap..$^{3-11}$ The ability of these systems to efficiently confine and enhance optical fields is crucial for applications, such as singlemolecule surface-enhanced Raman spectroscopy (SERS) ${ }^{12}$ or extreme-ultraviolet generation. ${ }^{7}$

There are many techniques to measure the near-field distributions in gap nanoantennas, such as scanning near-field optical microscopy ${ }^{9,11}$ and two-photon induced luminescence. ${ }^{4,8}$ However, these local probes have a limited spatial resolution of $10 \mathrm{~nm}$ at best. ${ }^{11}$ Thus, it would be highly desirable to develop new techniques or strategies that enable the extraction of information about the local fields in subnanometer metallic gaps, where the field enhancements are expected to be largest. Very recently, we made a step in this direction, ${ }^{13}$ investigating the electronic transport through atomic-scale gold electrodes separated by a subnanometer gap under near-infrared laser irradiation, finding that the irradiation induces a dc photocurrent. By comparing this photocurrent with low-frequency conduction measurements, we were able to determine the optical voltage generated across the gap and, in turn, to infer the electric field in this region. Enhancement factors exceeding $10^{3}$ were reported, in line with previous estimates from surface-enhanced Raman measurements. ${ }^{14-16}$ The goal of this Brief Report is to shed some light on the origin of this huge field enhancement in these atomic-scale gap antennas.

From a theoretical point of view, the field enhancement in finite metallic structures with nanometric gaps, such as nanoparticle or nanorod dimers, has been extensively studied (see Ref. 17 and references therein). However, here we are interested in the analysis of a sub-nanometer gap formed between two atomic-scale electrodes, thus coupled to semiinfinite leads, where, to our knowledge, no related studies have been reported. ${ }^{18,19}$ In this work, we present an analysis of the optical response of gold atomic junctions with subnanometer gaps based on finite difference time domain (FDTD) simulations. Our main findings are: (i) field enhancements exceeding $10^{3}$ are possible for gaps of a few angstrom, ${ }^{20}$ which supports the main conclusion of Ref. 13; (ii) the huge enhancements are due to the excitation of a hybrid plasmon involving large localized charge distributions of opposite sign on either side of the junction, in analogy with the dipolar bonding dimer plasmons found in nanoparticle dimers; ${ }^{21,22}$ and (iii) the plasmon resonances red shift as the interelectrode gap decreases, which also resembles the behavior found in nanoparticle ${ }^{21}$ and nanorod dimers. ${ }^{23}$

System and methodology. We model here the gold nanogaps of Ref. 13 with the idealized geometry of Fig. 1(a). In this geometry, two extended gold tips ending in hemispheres with a radius of $2 \mathrm{~nm}$ are separated by a distance $d$ (this distance is assumed to be the actual distance between the electron clouds). We also consider that the junction is placed in a vacuum and that there is no substrate. To analyze the optical response of this gold junction, we have performed FDTD simulations where the main features to be considered are: (i) the two tiplike electrodes are coupled to infinite metallic surfaces, which are described by perfect metallic boundary conditions; ${ }^{24}$ (ii) the structure is illuminated at normal incidence by a plane wave covering the whole region, and perfectly absorptive boundary conditions are placed on the illumination direction; (iii) the gold dielectric function used was extracted from ellipsometry measurements of a 20 -nm-thin film; ${ }^{25}$ and (iv) a nonuniform mesh with a smallest grid size of $0.25 \AA$ at the junction was used. All the simulations were performed with the code FDTD Solutions (from Lumerical Solutions, Inc., Canada).

Results and discussions. In Fig. 1(b), we show the spatial distribution of the electric-field intensity (normalized by the incident intensity) in the $x-z$ plane of a junction with $d=3 \AA$ illuminated by an incident light of $801 \mathrm{~nm}$ with its polarization along the junction axis ( $x$ axis). The field is strongly localized in the gap region, and the intensity (i.e., $|E|^{2}$ ) is enhanced by a factor larger than $6 \times 10^{5}$ in that region. We have systematically analyzed the field distribution as a function of both the interelectrode distance $d$ and the wavelength of the incident 
(a)

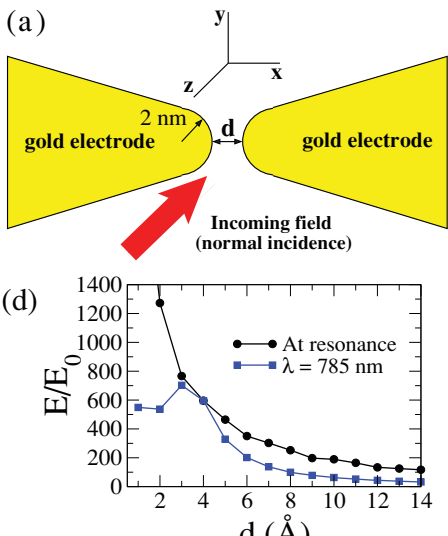

(b)

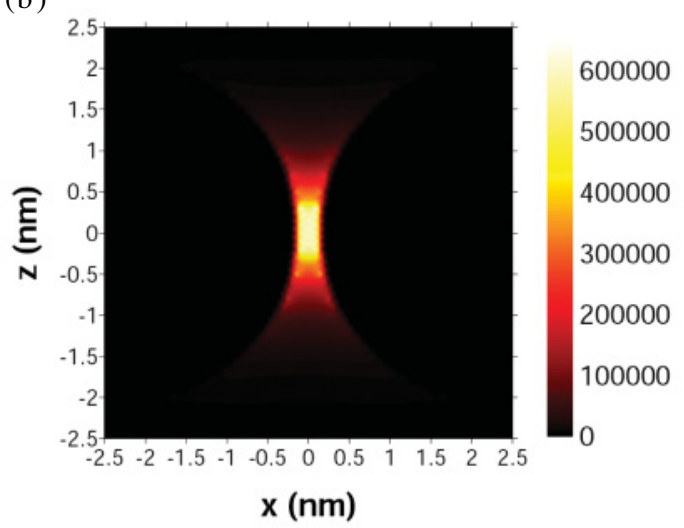

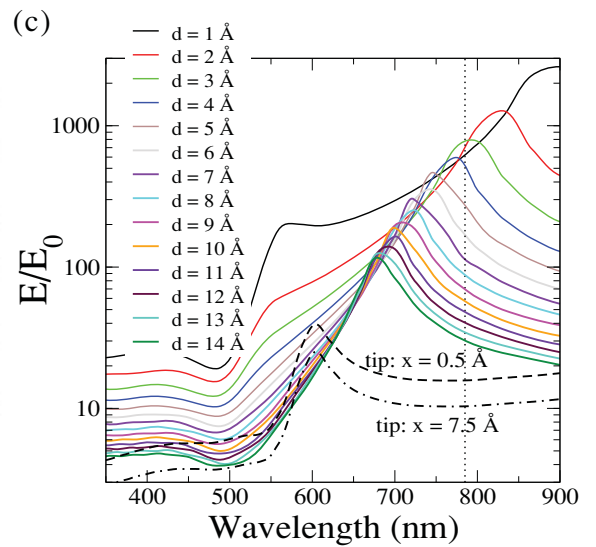

FIG. 1. (Color online) (a) Schematic representation of the gold junction considered in this work. (b) Spatial distribution of the field intensity (normalized by the incident one) in the $x-z$ plane for an interelectrode separation $d=3 \AA$. The wavelength of the illuminating light is $\lambda=801 \mathrm{~nm}$. (c) Field enhancement $\left(E / E_{0}\right)$ evaluated in the middle of the gap as a function of the wavelength of the incident light for different interelectrode separation, from $d=1 \AA$ (top) to $d=14 \AA$ (bottom). In all cases, the polarization is directed along the $x$ axis (junction axis). The dashed and dashed-dotted lines are the corresponding results for a single tip at distances $0.5 \AA$ and $7.5 \AA$ from the tip along the $x$ axis, respectively. The vertical dotted line indicates the wavelength $785 \mathrm{~nm}$ used in Ref. 13. (d) Field enhancement in the middle of the gap as a function of the interelectrode distance at resonance and at $\lambda=785 \mathrm{~nm}$.

light. A summary of the results can be seen in Fig. 1(c), where we show the field enhancement factor $\left(E / E_{0}\right)$ in the middle of the gap on the junction axis, with $E_{0}$ the amplitude of the incident field, as a function of the wavelength, and for different values of $d$ ranging from 1 to $14 \AA$. Notice the appearance of a resonance that shifts monotonically to the red as $d$ decreases. At large separations, this resonance wavelength tends toward that exhibited by a single tip, around $600 \mathrm{~nm}$ (see dashed and dashed-dotted lines). On the other hand, the enhancement factor on resonance reaches values larger than $10^{3}$ for $d<3 \AA$, which supports the estimates reported in Ref. 13.

The resonance seen in Fig. 1(c) clearly suggests that the incident light is exciting a plasmon-type mode. The comparison of the wavelength of that resonance with the one of a single tip and the fact that the resonance red shifts as $d$ decreases both indicate that this mode can be considered as a hybrid plasmon involving charge density oscillations of opposite signs on both sides of the junction. The red shift is a simple consequence of the increasing interelectrode interaction as $d$ decreases, which in turn leads to a reinforcement of the electric field in the gap region. It is worth stressing that in this case, the hybridization occurs between the continua of delocalized plasmon modes of the extended tips, rather than between localized plasmons as in the case of subwavelength nanoparticles (see discussion below).

The actual value of the field enhancement factor depends on the tip radius, and it increases monotonically as the radius decreases. This is precisely the well-known "lightning-rod" effect, see e.g., Ref. 26. In this case, the lightning-rod effect cooperates with the plasmon excitation to greatly enhance the field locally at the region. To get an idea of the impact of this effect, we have repeated the calculations with a smaller tip radius of $1.5 \AA$ and found that, while the spectral response is almost identical, the field maximum increases by approximately a $16 \%$.

In Ref. 13, it was found that the enhancement decreases slowly with $d$ (slower than $1 / d$ ). Our simulations show that this decay depends critically on the wavelength of the incident light. In Fig. 1(d), we show the enhancement factor in the middle of the gap as a function of $d$ both for the resonant wavelength and for $785 \mathrm{~nm}$, the wavelength used in Ref. 13. Notice that for the resonant condition, the field decays monotonically with the gap size approximately as $1 / d^{1.24}$, while for $\lambda=785 \mathrm{~nm}$, the field does not decay for very short distances, and for $d>4 \AA$, decays slightly faster than $1 / d^{2}$.

The results described above qualitatively resemble the predicted and observed optical response of nanoparticle dimers (see e.g., Ref. 21) and nanorods dimers (see e.g., Ref. 23). For instance, it is well known that when two nanoparticles are placed next to each other, the plasmon modes of the individual nanoparticles interact, resulting in hybridized dimer plasmon modes whose energies can be strongly red shifted with respect to that of the plasmon modes of the individual nanoparticles. This is essentially what happens in the gold junctions considered here. One may then wonder to what extent the field enhancement in gold junctions can be explained in terms of the physics of nanoparticle dimers. To answer this question, we have considered a dimer comprising two gold spheres of radius $2 \mathrm{~nm}$, which is the radius of the outermost part of the gold tips. ${ }^{27}$ In the upper panels of Fig. 2, we compare the field distributions for a gold junction with $d=6 \AA$ [panel (a)] and a dimer with the same separation [panel (b)]. In each case, the wavelength considered is the one that gives rise to the maximum field $(750 \mathrm{~nm}$ for the junction and $539 \mathrm{~nm}$ for the dimer). Notice that while the symmetry of the field distribution in the gap region is practically identical in both cases, there is a big difference in the magnitude of the field enhancements (of more than an order of magnitude). Moreover, as we show in Figs. 2(c) and 2(d), there is also an important difference in the spectral response, where the resonance for the extended electrode junction is considerably red shifted as compared with the dimer case. The smaller field enhancement for the dimer is mainly due to its smaller scattering cross section. Of course, in the dimer configuration, one could reach the 

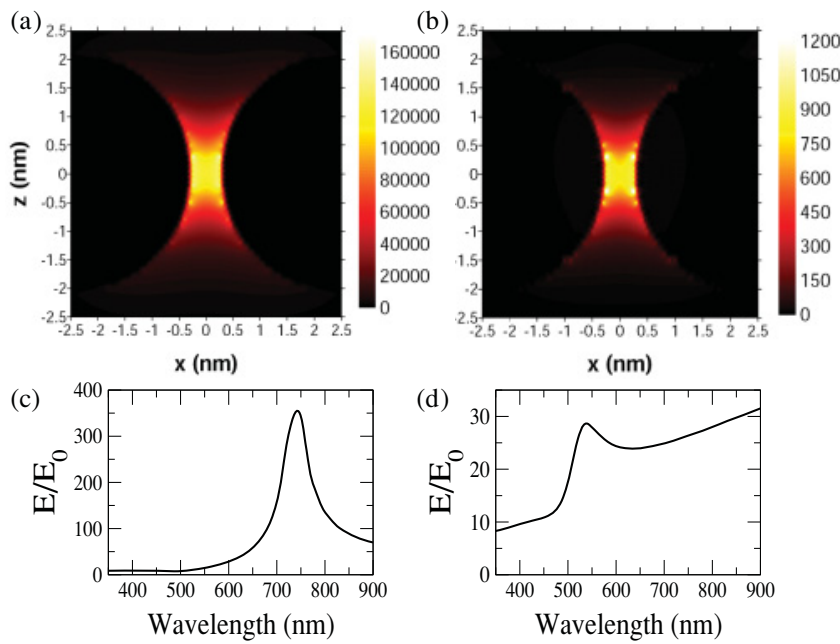

FIG. 2. (Color online) (a) Spatial distribution of the normalized field intensity in the gold junction for $d=6 \AA$ and $\lambda=750 \mathrm{~nm}$. (b) The same as in panel (a), but for two spheres of radius $2 \mathrm{~nm}$ separated $6 \AA$ and illuminated with light of $539 \mathrm{~nm}$. (c) Field enhancement $\left(E / E_{0}\right)$ evaluated in the middle of the gap of the gold junction of panel (a) as a function of the light wavelength. (d) The same as in panel (c), but for the nanoparticle dimer of panel (b).

enhancement factors of the junction for the same gap size, but that would require the nanoparticles to have a considerably larger radius, ${ }^{28}$ which would be unrealistic for an atomic-size contact. Although less important, the difference in the field values reached at the resonant conditions in both structures is also partly due to the frequency dependence of the gold permittivity, which in particular has a slightly larger imaginary part at the frequency of the plasmon resonance of the dimer. ${ }^{29}$

The plasmonic origin of the field enhancement suggests that it should be very sensitive to the polarization of the incident field. ${ }^{30}$ This is indeed the case, as we illustrate in Fig. 3. This figure shows the intensity distribution in a junction with electrode separation $d=6 \AA$ and $\lambda=495 \mathrm{~nm}$, but this time, the polarization is directed along the axis perpendicular to the

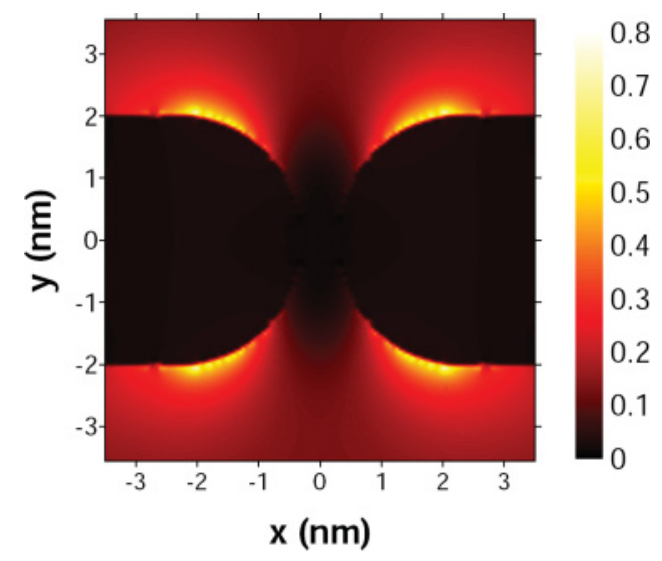

FIG. 3. (Color online) Spatial distribution of the normalized field intensity in the $x-y$ plane for a gold junction with $d=6 \AA$ and illuminated with light of $495 \mathrm{~nm}$ with its polarization directed along the axis perpendicular to the junction axis ( $y$ axis). The wavelength used is the one at which the field reaches its maximum in the middle of the junction. junction axis [this figure has to be compared with Fig. 2(a)]. As one can see, the field distribution is now quite different (with the charge oscillating back and forth in the transversal direction) and, in particular, the near field is smaller than the incident field everywhere in the junction region. Notice that a field distribution like this does not generate an optical voltage across the junction, and, therefore, there would not be any photocurrent in a transport experiment like the one of Ref. 13. We have also verified that the strong polarization dependence of the field enhancement persists even in very asymmetric contacts with a pronounced misalignment of the axes of the tips. Further investigation into other tip geometries are ongoing.

It is important to discuss the limitations of our classical theory. First, we have assumed that the optical response of these nanocontacts is well described by a classical frequencydependent dielectric function. However, non local effects can play some role in the outermost part of the electrodes, when the dimensions are smaller than the mean free path of the valence electrons that participate in the plasmon resonances. ${ }^{31}$ Second, the onset of quantum effects when the tips are very close can be much more important. As discussed in Ref. 32, the onset of quantum tunneling and the concomitant charge transfer between the electrodes can lead to the appearance of a charge transfer plasmon involving conduction electrons flowing back and forth between the metallic tips. A finite electron density between the tips could give rise to a screening of the plasmonic interactions responsible for the strong red shift of the dipolar plasmons and, in turn, could reduce the field in the gap region. ${ }^{33}$ In any case, while our classical approach neglects detailed electronic structure, the essential physics of plasmon hybridization involving the continuum modes of the extended electrodes is expected to survive intact in realistic quantum-mechanical calculations.

Conclusions. We have studied the optical response of atomic-scale gold junctions with subnanometer gaps, within the framework of classical electromagnetism. We have shown that the huge field enhancements reported experimentally ${ }^{13}$ originate from the excitation of hybrid plasmons resonances involving charge oscillations in both electrodes. Such resonances red shift as the gap size decreases as a consequence of the increase of the interelectrode interaction. Our results on the magnitude of the field enhancement clearly indicate that metallic nanogaps can be ideal templates for surface-enhanced Raman scattering of single molecules, which are very important for both molecular electronics and sensing applications. ${ }^{34}$

Acknowledgments. We thank F. J. Garcia-Vidal, J. J. Sáenz, and J. K. Viljas for valuable discussions. A. G. M. acknowledges financial support from the EU (Grant No. NMP3SL-2008-214107-Nanomagma) and the Spanish MICINN ("MAGPLAS" Grant No. MAT2008-06765-C02-01/NAN and "FUNCOAT" CONSOLIDER INGENIO Grant No. 2010 CSD2008-00023). J. C. C. acknowledges support from the EU through the BIMORE network (Grant No. MRTN-CT2006-035859) and the Spanish MICINN (Grant No. FIS200804209). D. N. and D. R. W. acknowledge support from the Robert A. Welch Foundation (Grant No. C-1636) and the Lockheed Martin Advanced Nanotechnology Center of Excellence at Rice University (LANCER). 
${ }^{1}$ S. A. Maier, Plasmonics: Fundamentals and Applications (Springer, New York, 2007).

${ }^{2}$ J. A. Schuller, E. S. Barnard, W. Cai, Y. C. Jun, J. S. White, and M. L. Brongersma, Nat. Mater. 9, 193 (2010).

${ }^{3}$ R. D. Grober, R. J. Schoellkopf, and D. D. Prober, Appl. Phys. Lett. 70, 1354 (1997).

${ }^{4}$ P. Mühlschlegel, H. J. Eisler, O. J. F. Martin, B. Hecht, and D. W. Pohl, Science 308, 1607 (2005).

${ }^{5}$ P. J. Schuck, D. P. Fromm, A. Sundaramurthy, G. S. Kino, and W. E. Moerner, Phys. Rev. Lett. 94, 017402 (2005).

${ }^{6}$ L. Tang, S. E. Kocabas, S. Latif, A. K. Okyay, D.-S. Ly-Gagnon, K. C. Saraswat, and D. A. B. Miller, Nat. Photonics 2, 226 (2008).

${ }^{7}$ S. Kim, J. Jin, Y.-J. Kim, I.-Y. Park, Y. Kim, and S.-W. Kim, Nature (London) 453, 757 (2008).

${ }^{8}$ P. Ghenuche, S. Cherukulappurath, T. H. Taminiau, N. F. van Hulst, and R. Quidant, Phys. Rev. Lett. 101, 116805 (2008).

${ }^{9}$ M. Schnell, A. García-Etxarri, A. J. Huber, K. Crozier, J. Aizpurua, and R. Hillenbrand, Nat. Photonics 3, 287 (2009).

${ }^{10}$ For a recent review on optical antennas, see P. Bharadwaj, B. Deutsch, and L. Novotny, Adv. Opt. Photon. 1, 438 (2009).

${ }^{11}$ M. Schnell, A. García-Etxarri, J. Alkorta, J. Aizpurua, and R. Hillenbrand, Nano Lett. 10, 3524 (2010).

${ }^{12}$ H. Xu, E. J. Bjerneld, M. Käll, and L. Börjesson, Phys. Rev. Lett. 83, 4357 (1999).

${ }^{13}$ D. Ward, F. Hüser, F. Pauly, J. C. Cuevas, and D. Natelson, Nat. Nanotechnol. 5, 732 (2010).

${ }^{14}$ D. R. Ward, N. J. Halas, J. W. Ciszek, J. M. Tour, Y. Wu, P. Nordlander, and D. Natelson, Nano Lett. 7, 1396 (2007).

${ }^{15}$ D. R. Ward, N. K. Grady, C. S. Levin, N. J. Halas, Y. Wu, P. Nordlander, and D. Natelson, Nano Lett. 8, 919 (2008).

${ }^{16}$ D. R. Ward, G. D. Scott, Z. K. Keane, N. J. Halas, and D. Natelson, J. Phys. Condens. 20, 374118 (2008).

${ }^{17}$ M. Pelton, G. W. Bryant, and J. Aizpurua, Laser and Photon. Rev. 2, 136 (2008).

${ }^{18}$ The field enhancement in individual metallic tips has been extensively studied in the context of optical tweezers and local probe microscopes, see e.g., L. Novotny, R. X. Bian, and X. S. Xie,
Phys. Rev. Lett. 79, 645 (1997); O. J. F. Martin and C. Girard, Appl. Phys. Lett. 70, 705 (1997).

${ }^{19}$ In Ref. 15, the optical properties of gold nanojunctions were studied, but all characteristic dimensions of the system were above $1 \mathrm{~nm}$.

${ }^{20}$ Enhancements larger than $10^{3}$ in self-similar chains of metal nanospheres were predicted in K. Li, M. I. Stockman, and D. J. Bergman, Phys. Rev. Lett. 91, 227402 (2003).

${ }^{21}$ I. Romero, J. Aizpurua, G. W. Bryant, and F. J. García de Abajo, Opt. Express 14, 9988 (2006).

${ }^{22}$ O. Pérez-González, N. Zabala, A. G. Borisov, N. J. Halas, P. Nordlander, and J. Aizpurua, Nano Lett. 10, 3090 (2010).

${ }^{23}$ J. Aizpurua, G. W. Bryant, L. J. Richter, F. J. García de Abajo, B. K. Kelley, and T. Mallouk, Phys. Rev. B 71, 235420 (2005).

${ }^{24}$ The infinite surfaces are placed $8 \mathrm{~nm}$ away from the center of the junction.

${ }^{25}$ E. Ferreiro-Vila, J. B. González-Díaz, R. Fermento, M. U. González, A. García-Martín, J. M. García-Martín, A. Cebollada, G. Armelles, D. Meneses-Rodríguez, and E. M. Sandoval, Phys. Rev. B 80, 125132 (2009).

${ }^{26}$ J. Gersten and A. Nitzan, J. Chem. Phys. 73, 3023 (1980).

${ }^{27}$ For the description of the nanoparticle dimer, we replaced the infinite metallic surfaces used for the junction by perfectly absorptive boundary conditions.

${ }^{28}$ A. Aubry, D. Y. Lei, S. A. Maier, and J. B. Pendry, Phys. Rev. Lett. 105, 233901 (2010).

${ }^{29}$ The gold dielectric constants in our case are $\epsilon=-6.28+2.04 i$ for $\lambda=539 \mathrm{~nm}$ and $\epsilon=-23.46+1.63 i$ for $\lambda=750 \mathrm{~nm}$.

${ }^{30}$ J.-H. Tian, B. Liu, X. Li, Z.-L. Yang, B. Ren, S.-T. Wu, N. Tao, and Z.-Q. Tian, J. Am. Chem. Soc. 128, 14748 (2006).

${ }^{31}$ R. Fuchs and F. Claro, Phys. Rev. B 35, 3722 (1987).

${ }^{32}$ J. Zuloaga, E. Prodan, and P. Nordlander, Nano Lett. 9, 887 (2009).

${ }^{33}$ L. Mao, Z. Li, B. Wu, and H. Xu, Appl. Phys. Lett. 94, 243102 (2009).

${ }^{34}$ See e.g., J. C. Cuevas and E. Scheer, Molecular Electronics: An Introduction to Theory and Experiment (World Scientific, Singapore, 2010), Chap. 20. 